\title{
AN APPLICATION OF BANACH LIMITS
}

\author{
Z. U. AHMAD AND MURSALEEN
}

(Communicated by R. Daniel Mauldin)

\begin{abstract}
Let $l_{\infty}$ denote the Banach space of bounded sequences, $\sigma$ an injection of the set of positive integers into itself having no finite orbits, and $T$ the operator defined on $l_{\infty}$ by $T y(n)=y(\sigma n)$. A positive linear functional $\mathscr{L}$ with $\|\mathscr{L}\|=1$, is called a $\sigma$-mean if $\mathscr{L}(y)=\mathscr{L}(T y)$ for all $y$ in $l_{\infty}$. A sequence $y$ is said to be $\sigma$-convergent, denoted $y \in V_{\sigma}$, if $\mathscr{L}(y)$ takes the same value, called $\sigma-\lim y$, for all $\sigma$-means $\mathscr{L}$. P. Schaefer [6] gave necessary and sufficient conditions on a matrix $A$ to ensure that $A(c) \subset V_{\sigma}$, where $c$ is the space of convergent sequences, and additional conditions ensuring that $\sigma$ - $\lim A y=\lim y$ for all $y \in c$, denoting the class of matrices satisfying these conditions by $\left(c, V_{\sigma}\right)_{1}$ and calling them the $\sigma$-regular matrices. In this paper, we use such matrices to find the sum of a sequence of Walsh functions.
\end{abstract}

1. Introduction. Let $l_{\infty}$ and $c$ be the Banach spaces of bounded and convergent sequences, respectively, with usual norm $\|y\|=\sup _{k}\left|y_{k}\right|$, and let $\mathrm{BV}$ be the Banach space of sequences of bounded variation normed by $\|y\|=\sum_{k}\left|y_{k}-y_{k-1}\right|$. We denote by $V_{\sigma}$, the set of bounded sequences all of whose invariant means are equal. In case $\sigma$ is the translation mapping $n \rightarrow n+1$, a $\sigma$-mean is often called a Banach limit and $V_{\sigma}$ is the set of almost convergent sequences [3]. A $\sigma$-mean extends the limit functional on $c$ in the sense that $\mathscr{L}(y)=\lim y$ for all $y \in c$ if and only if $\sigma$ has no finite orbits (see [4 and 5]).

Schaefer [6] gave the following necessary and sufficient conditions for an infinite matrix $A=\left(a_{n k}\right)$ to be $\sigma$-regular:

$$
\begin{gathered}
\|A\|=\sup _{n} \sum_{k}\left|a_{n k}\right|<+\infty \\
a_{(k)}=\left\{a_{n k}\right\}_{n=1}^{\infty} \in V_{\sigma} \text { with } \sigma \text {-limit zero for each } k,
\end{gathered}
$$

and

$$
a=\left\{\sum_{k} a_{n k}\right\}_{n=1}^{\infty} \in V_{\sigma} \quad \text { with } \sigma \text {-limit }+1 .
$$

In this paper, we use such $\sigma$-regular matrices to sum the following sequence of Walsh functions. Let us define a sequence of functions $h_{0}(x), h_{1}(x), \ldots, h_{n}(x)$ which satisfy the following conditions:

$$
h_{0}(x)= \begin{cases}1, & 0 \leq x<\frac{1}{2} \\ -1, & \frac{1}{2} \leq x<1\end{cases}
$$

Received by the editors March 10, 1987.

1980 Mathematics Subject Classification (1985 Revision). Primary 40C05; Secondary 42C10.

Key words and phrases. Walsh functions, Banach limits, infinite matrices. 
$h_{0}(x+1)=h_{0}(x)$, and $h_{n}(x)=h_{0}\left(2^{n} x\right), n=1,2,3, \ldots$ The functions $h_{n}(x)$ are called Rademacher's functions.

The Walsh functions are defined by

$$
\begin{aligned}
& \phi_{0}(x)=1 \\
& \phi_{n}(x)=h_{n_{1}}(x) h_{n_{2}}(x) \cdots h_{n_{r}}(x), \quad 0 \leq x \leq 1,
\end{aligned}
$$

for $n=2^{n_{1}}+2^{n_{2}}+\cdots+2^{n_{r}}$, where the integers $n_{i}$ are uniquely determined by $n_{i+1}<n_{i}$.

Let $f$ be $L$-integrable and periodic with period 1 , and let the Walsh-Fourier series of $f$ be

where

$$
\sum_{n=1}^{\infty} a_{n} \phi_{n}(x)
$$

$$
a_{n}=\int_{0}^{1} f(x) \phi_{n}(x) d x
$$

are called the Walsh-Fourier coefficients of $f$.

We recall some basic properties of Walsh functions (see [2]): For each fixed $x$ and for all $t$,

(i) $\phi_{n}(x+t)=\phi_{n}(x) \phi_{n}(t)$,

(ii) $\int_{0}^{1} f(x+t) d t=\int_{0}^{1} f(t) d t$, and

(iii) $\int_{0}^{1} f(t) \phi_{n}(x+t) d t=\int_{0}^{1} f(x+t) \phi_{n}(t) d t$,

where $\dot{+}$ denotes the operation in the dyadic group, the set of all sequences $\bar{s}=\left\{s_{n}\right\}$, $s_{n}=0,1$ for $n=1,2,3, \ldots$, is addition modulo 2 in each coordinate.

Let

$$
J_{k}(x)=\int_{0}^{x} \phi_{k}(t) d t, \quad k=0,1,2, \ldots
$$

It is easy to see that $J_{k}(x)=0$ for $x=0,1$.

2. The main result. We establish the following Theorem concerning $\sigma$-regular matrices.

THEOREM. Let $z_{x}$ be the sequence defined by $z_{x}(k)=k J_{k}(x)$. Let $s_{x}$ be the sequence defined by $s_{x}(k)=k a_{k} \phi_{k}(x)$ for an L-integrable function $f$. Let $A$ be a $\sigma$-regular matrix. Then the following statements about $A$ are equivalent:

(2.1) $A s_{x} \in V_{\sigma}$ with $\sigma$-limit zero, whenever $x$ is a point at which $f(x) \in \mathrm{BV}$.

(2.2) $A z_{x} \in V_{\sigma}$ and has $\sigma$-limit zero for all $x$.

PrOOF. We have

$$
\begin{aligned}
s_{x}(k) & =k a_{k} \phi_{k}(x) \\
& =k \int_{0}^{1} f(t) \phi_{k}(t) \phi_{k}(x) d t \\
& =k \int_{0}^{1} f(t) \phi_{k}(x+t) d t \\
& =k \int_{0}^{1} f(x+t) \phi_{k}(t) d t \\
& =k\left[f(x+t) J_{k}(t)\right]_{0}^{1}-\int_{0}^{1} z_{t}(k) d f(x+t) .
\end{aligned}
$$


Since $k\left[f(x \dot{+} t) J_{k}(t)\right]_{0}^{1}=0$ by the fact that $J_{k}(1)=J_{k}(0)=0$. Therefore, for every positive integer $n$,

$$
\sum_{k=1}^{\infty} \sum_{j=0}^{p} \frac{a\left(\sigma^{j}(n), k\right) s_{x}(k)}{p+1}=-\int_{0}^{1} B_{p, n}(t) d h_{x}(t)
$$

where

$$
B_{p, n}(t)=\sum_{k=1}^{\infty} \sum_{j=0}^{p} \frac{a\left(\sigma^{j}(n), k\right)}{p+1} z_{t}(k)
$$

and

$$
h_{x}(t)=f(x+t) .
$$

Now, it is sufficient to show that $\int_{0}^{1} B_{p, n}(t) d h_{x}(t) \rightarrow 0$, as $p \rightarrow \infty$, uniformly in $n$. Since, the Banach weak convergence theorem [1] states that $\int_{0}^{1} g_{n} d h_{x} \rightarrow 0$ for all $h_{x} \in \mathrm{BV}$ if and only if $\left\|g_{n}\right\|<M$ for all $n$ and $g_{n} \rightarrow 0$ pointwise. Therefore, if we choose $g_{n}=\left\{B_{p, n}(t)\right\}$, i.e. the sequence of the $A$-transforms of the particular sequence $z=\left\{k J_{k}(t)\right\}$ for any $t \in R$, and it says, in brief, that if $A$ is a $\sigma$-regular matrix method, then $\left\|A z_{t}\right\| \leq\|A\|$, where $\|A\|$ is the norm of $A$ regarded as a linear mapping from the Banach space $c$ to the Banach space $l_{\infty}$. But this is immediate once it is observed that $A$ also maps $l_{\infty}$ to $l_{\infty}$, with the same norm, as is true of any method satisfying (1.1).

This completes the proof.

\section{REFERENCES}

1. S. Banach, Theorie des operations lineaires, PWN, Warszawa, 1932.

2. N. J. Fine, On Walsh functions, Trans. Amer. Math. Soc. 65 (1949), 372-414.

3. G. G. Lorentz, A contribution to the theory of divergent sequences, Acta Math. 80 (1948), 167-190.

4. Mursaleen, On some new invariant matrix methods of summability, Quart. J. Math. Oxford (2) 34 (1983), 77-86.

5. R. A. Raimi, Invariant means and invariant matrix methods of summability, Duke Math. J. 30 (1963), 81-94.

6. P. Schaefer, Infinite matrices and invariant means, Proc. Amer. Math. Soc. 36 (1972), 104-110.

Department of Mathematics, Aligarh Muslim University, Aligarh 202001, INDIA

Department of Applied Mathematics, Z. H. College of Engineering and Technology, Aligarh Muslim University, Aligarh 202001, India 